\title{
Computational characterization of ignition regimes in a syngas/air mixture with temperature fluctuations
}

\author{
Pinaki Pal ${ }^{\mathrm{a}}$, Mauro Valorani ${ }^{\mathrm{b}}$, Paul G. Arias ${ }^{\mathrm{a}, \mathrm{c}}$, Hong G. Im ${ }^{\mathrm{c}, *}$, Margaret S. Wooldridge ${ }^{\mathrm{a}, \mathrm{d}}$, \\ Pietro P. Ciottoli ${ }^{\mathrm{b}}$, Riccardo M. Galassi ${ }^{\mathrm{b}}$
}

\begin{abstract}
Auto-ignition characteristics of compositionally homogeneous reactant mixtures in the presence of thermal non-uniformities and turbulent velocity fluctuations were computationally investigated. The main objectives were to quantify the observed ignition characteristics and numerically validate the theory of the turbulent ignition regime diagram recently proposed by Im et al. [H.G. Im, P. Pal, M.S. Wooldridge, A.B. Mansfield, Combustion Science and Technology, 2015] that provides a framework to predict ignition behavior a priori based on the thermo-chemical properties of the reactant mixture and initial flow and scalar field conditions. Ignition regimes were classified into three categories: weak (where deflagration is the dominant mode of fuel consumption), reaction-dominant strong, and mixing-dominant strong (where volumetric ignition is the dominant mode of fuel consumption). Two-dimensional (2D) direct numerical simulations (DNS) of auto-ignition in a lean syngas/air mixture with uniform mixture composition at highpressure, low-temperature conditions were performed in a fixed volume. The initial conditions considered two-dimensional isotropic velocity spectrums, temperature fluctuations and localized thermal hot spots. A number of parametric test cases, by varying the characteristic turbulent Damköhler and Reynolds numbers, were investigated. The evolution of the auto-ignition phenomena, pressure rise, and heat release rate were analyzed. In addition, combustion mode analysis based on front propagation speed and computational singular perturbation (CSP) was applied to characterize the auto-ignition phenomena. All results supported that the observed ignition behaviors were consistent with the expected ignition regimes predicted by the theory of the regime diagram. This work provides new high-fidelity data on syngas ignition characteristics over a broad range of conditions and demonstrates that the regime diagram serves as a predictive guidance in the understanding of various physical and chemical mechanisms controlling auto-ignition in thermally inhomogeneous and compositionally homogeneous turbulent reacting flows.
\end{abstract}

Keywords: Direct numerical simulation, strong and weak ignition, ignition regimes, temperature fluctuations, computational singular perturbation 


\section{Introduction}

Low-temperature combustion (LTC) strategies have the potential to improve efficiency and reduce $\mathrm{NO}_{\mathrm{x}}$ emissions in both transportation [1] and stationary power devices [2]. Reductions in combustion temperature can be achieved by operating at lean, nearly homogeneous, and/or diluted, conditions. However, issues related to combustion stability, safety and control may arise at these conditions, due to increased influence of abnormal ignition behaviors such as early ignition and flashback in gas turbines [2,3] or knock in reciprocating engines [4]. Improved understanding and better prediction of auto-ignition characteristics are therefore valuable for successful implementation of these advanced technologies.

Many experimental studies have been conducted to investigate auto-ignition of different fuels such as hydrogen [5-9], iso-octane [10,11] and syngas [12, 13], at conditions relevant to practical combustion systems. Two types of auto-ignition regimes were commonly observed: strong (spatially homogeneous ignition) and weak (localized reaction sites and deflagration). Moreover, the boundary between strong and weak ignition regimes, known as the strong ignition limit, was found to coincide with an iso-line of the sensitivity of homogeneous ignition delay time to temperature, $d \tau_{i g} / d T$, in the pressure-temperature space $[6,13]$. Recent experimental investigations of syngas auto-ignition [14-18] at conditions relevant to gas turbine operation reported large discrepancies between measurements and homogeneous chemical kinetic modeling predictions of ignition delay times, with the former being orders of magnitude lower than the latter. Mansfield and Wooldridge [13] studied syngas auto-ignition in a rapid compression facility and demonstrated that the discrepancy was due to transition from strong to weak ignition regime as the initial mean temperature was lowered. In addition, a simple criterion proposed by Sankaran et al. [19] (referred to as the Sankaran criterion here), based on Zel'dovich's original theory [20] was found to capture the experimentally observed strong ignition limit a priori. This predictive criterion is defined as the ratio of laminar flame speed to the thermal gradient driven spontaneous propagation speed. Pal et al. [21] further numerically validated the Sankaran criterion as a 
predictive indicator of the ignition regime for homogeneous mixtures in the presence of thermal non-uniformities through parametric studies of syngas auto-ignition in a one-dimensional configuration.

In addition to thermal inhomogeneities, the presence of turbulence may also significantly influence auto-ignition phenomena in practical devices. Ihme and co-workers [22, 23] demonstrated using a reduced order modeling approach that turbulent fluctuations could result in significant advancement of overall ignition. A number of DNS studies [24-28] have revealed that thermal stratification and turbulence can influence auto-ignition phenomena in LTC engines. More recently, Im et al. [29] conducted a theoretical scaling analysis and proposed nondimensional criteria in terms of the characteristic Damköhler and Reynolds numbers of a system to predict the occurrence of strong and weak ignition regimes in thermally inhomogeneous turbulent reacting flows. The criteria formulated by extending the original Sankaran criterion [19] and taking into account the effects of passive scalar mixing due to turbulence, ultimately led to the development of a turbulent ignition regime diagram [29]. An alternative version of ignition regime diagram has also been proposed by Grogan et al. [30].

The present computational study investigates the effects of thermal inhomogeneities and turbulence on syngas auto-ignition behaviour. 2D DNS of auto-ignition in a lean syngas/air mixture are performed at various parametric conditions. The high-fidelity simulations aim to provide additional insight into the range of ignition behaviors that can be expected under highpressure low-temperature conditions, and also provide numerical validation of the turbulent ignition regime diagram [29]. In the next section, the ignition regime diagram is briefly reviewed. The numerical setup for simulations is presented next. The auto-ignition behaviors are subsequently characterized and the results are discussed in the context of the corresponding predictions of the regime diagram. 


\section{Turbulent ignition regime diagram}

One of the non-dimensional parameters used as an ignition regime criterion is the Sankaran number, Sa [19]:

$$
\mathrm{Sa} \approx \beta S_{L}\left|\frac{d \tau_{i g}}{d T}\right| \widetilde{\nabla T} \mid
$$

where $S_{L}$ and $\tau_{i g}$ are the laminar flame speed and homogeneous ignition delay corresponding to

the initial bulk mixture conditions, $|\widetilde{\nabla T}|$ denotes the statistical mean temperature gradient [29] and $\beta$ is a constant of order unity. In the present study, $\beta$ is equal to 0.5 [19]. Scaling analysis yields the following expression for Sa [29]:

$$
\mathrm{Sa}=K \mathrm{Da}_{\ell}{ }^{1 / 2}, K=\left(\frac{T^{\prime}}{\left(f_{i g}\right)^{1 / 2}}\right)\left|\frac{d_{i g}}{d T}\right|
$$

Here, $f=/ S_{L}^{2}$ is the flame time scale, where $\alpha$ is the thermal diffusivity of the initial bulk reactant mixture. $T$ represents the magnitude of thermal fluctuation. $\mathrm{Da}_{\ell}={ }_{t}{ }_{i}$ ig is the integral scale Damköhler number, where $\tau_{t}$ is the turbulence time scale defined as the ratio of integral length scale $(\ell)$ to velocity fluctuation $(u) . K$ is referred to as the normalized thermal ignition sensitivity [29].

The second ignition regime criterion is based on mixing Damköhler number, Da , defined as the ratio of time scale associated with the Taylor microscale ( ) to ${ }_{i g}$ : 


$$
\mathrm{Da}=\frac{\ell}{\mathrm{ig}}=\frac{\ell}{\mathrm{ig} \quad \ell}=\mathrm{Da}_{\ell} \operatorname{Re}_{\ell}^{1 / 3}
$$

where, $\operatorname{Re}_{\ell}=u \ell /$ is the turbulent Reynolds number, and is the kinematic viscosity of the bulk mixture gas.

Weak (W) ignition is expected if $\mathrm{Sa}>1$, and strong ignition is expected if $\mathrm{Sa}<1$ [19, 21$]$. Therefore, Eq. (2) suggests that weak ignition is possible for $\mathrm{Da}_{\ell}<K^{2}$. If $\mathrm{Da}_{\ell}>K^{2}$, the reactant mixture ignites spontaneously despite some level of temperature fluctuations. This is referred to as the reaction-dominant strong (RD-S) ignition regime. In addition, $\mathrm{Da}_{\ell}<1$ indicates that turbulent mixing is sufficiently rapid and the temperature fluctuations will be dissipated before local ignition kernels are formed. This is referred to as the mixing-dominant strong (MDS) ignition regime. The conditions between $\mathrm{Da}<1$ and $\mathrm{Da}_{\ell}>1$ represent a "grey" zone, in that some mixed mode ignition may occur. This is denoted as the mixing dominant or mixed/strong (MXD) ignition regime. These different ignition criteria lead to the regime diagram as shown in Fig. 1.

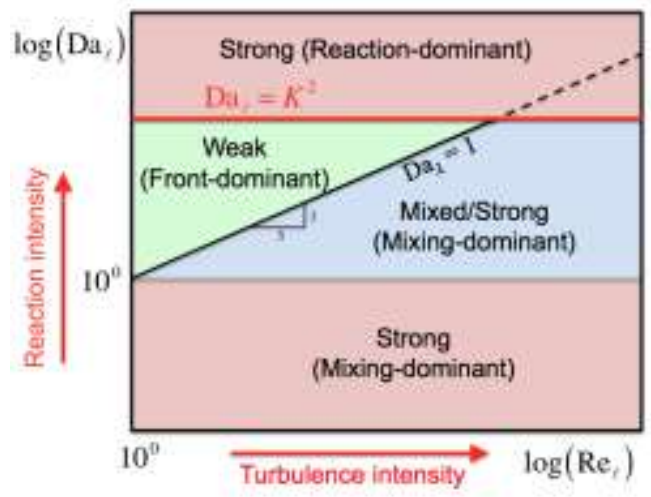

Fig. 1. Regime diagram for auto-ignition of homogeneous reactant mixture with temperature fluctuations and turbulence [29]. 


\section{Numerical method and initial conditions}

2D simulations were performed using the DNS code, S3D [31], which solves the compressible, Navier-Stokes, species and energy equations using a fourth-order explicit RungeKutta method for time integration and an eighth-order central differencing scheme for spatial discretization [32]. A detailed $\mathrm{H}_{2} / \mathrm{CO}$ mechanism with 12 species and 33 chemical reactions from Li et al. [33] was linked with CHEMKIN [34] and TRANSPORT [35] libraries for evaluating the reaction rates and thermodynamic and mixture-averaged transport properties, respectively. Periodic boundary conditions were imposed in all directions to represent constant volume ignition.

A uniform syngas/air reactant mixture of equivalence ratio of $0.5, \mathrm{H}_{2}: \mathrm{CO}$ molar ratio of 0.7:1, an initial pressure of 20 atm, was chosen. In addition, the mixture was diluted with excess nitrogen equal to twice the amount of nitrogen present in the air, resulting in the overall molar ratio of $\mathrm{N}_{2}: \mathrm{O}_{2}=11.28$ in the reactant mixture, to ensure the pressure rise was sufficiently high to serve as an indicator of auto-ignition, but also to avoid shock wave formation [36, 37], which cannot be captured by the employed computational schemes at present.

Turbulent velocity fluctuations were superimposed on a stationary mean velocity field based on an isotropic kinetic energy spectrum function [38]. A similar random temperature spectrum, uncorrelated with the turbulent kinetic energy spectrum, was superimposed on a constant mean temperature field. In addition, a thermal hot spot was superimposed on the temperature field in the form [19]:

$$
T_{h s}(x, y)=-\frac{A}{\exp }\left[\frac{2 n^{2}\left((x \quad L / 2)^{2}+(\mathrm{y} L / 2)^{2}\right.}{L^{2}}\right] \frac{A}{2 n^{2}}
$$

where $L$ is the length of each side of a $2 \mathrm{D}$ square box domain, $A=450, n=12$ and $(x, y)$ represents spatial location. The peak temperature of the hot spot was approximately $100 \mathrm{~K}$, which 
is the upper limit of the local temperature fluctuations commonly observed in ignition experiments [39].

A total of eight DNS cases A-H were considered by varying initial mean temperature $\left(T_{0}\right)$, most energetic turbulent length scale $\left(\ell_{e}\right)$ and $u$. For the four different $T_{0}$ values of 1100, 1020, 990 and $970 \mathrm{~K}$ considered, the computed $S_{L}$ values from PREMIX [40] simulations were 67.7, 27.8, 20.5, and $16.5 \mathrm{~cm} / \mathrm{s}$, respectively. $L=1.075 \mathrm{~cm}$ and $1.5 \mathrm{~cm}$ for cases A-E and F-H, respectively. $T$ was fixed at $15 \mathrm{~K}$ for all cases. The turbulence time scale was defined by ${ }_{t}=\ell_{e} / u$. The most energetic length scales of velocity and the temperature fluctuations were the same for all cases. For all simulations, the Kolmogorov length scale was resolved with at least 1 grid point [41] and the thinnest reaction fronts were resolved with at least 10 grid points. Details of the physical parameters for the different cases and the expected ignition regimes are listed in Table 1. All the parametric cases are plotted on the regime diagram in Fig. 2. The initial temperature profile for case A is shown in Fig. 3.

Table 1

Physical and regime diagram parameters for the DNS cases.

\begin{tabular}{|c|c|c|c|c|c|c|c|c|c|c|}
\hline Case & $T_{0}(\mathrm{~K})$ & $\begin{array}{c}i g \\
(\mathrm{~ms})\end{array}$ & $K^{2}$ & $\begin{array}{c}\ell_{e} \\
(\mathrm{~mm})\end{array}$ & $\begin{array}{c}u \\
(\mathrm{~m} / \mathrm{s})\end{array}$ & $\begin{array}{c}t \\
(\mathrm{~ms})\end{array}$ & $\mathrm{Da}_{\ell}$ & $\operatorname{Re}_{\ell}$ & $\mathrm{Da}$ & $\begin{array}{l}\text { Ignition } \\
\text { Regime }^{\mathrm{a}}\end{array}$ \\
\hline A & 990 & 25.77 & 4.05 & 4.3 & 0.05 & 86.0 & 3.34 & 35.24 & 1.02 & $\mathrm{~W}$ \\
\hline B & 1100 & 2.07 & 2.51 & 4.3 & 0.05 & 86.0 & 41.6 & 29.40 & 13.5 & RD-S \\
\hline $\mathrm{C}$ & 990 & 25.77 & 4.05 & 4.3 & 1.50 & 2.87 & 0.11 & 1057.4 & 0.01 & MD-S \\
\hline $\mathrm{D}$ & 1100 & 2.07 & 2.51 & 1.4 & 0.325 & 4.31 & 2.08 & 62.2 & 0.6 & MXD \\
\hline$E$ & 1020 & 12.7 & 3.28 & 4.0 & 0.3 & 13.33 & 1.05 & 185.0 & 0.2 & MXD \\
\hline $\mathrm{F}$ & 1100 & 2.07 & 2.51 & 6.0 & 0.2 & 30.0 & 14.5 & 164.0 & 2.65 & RD-S \\
\hline G & 990 & 25.77 & 4.05 & 6.0 & 0.2 & 30.0 & 1.16 & 197.0 & 0.2 & MXD \\
\hline $\mathrm{H}$ & 970 & 41.26 & 4.41 & 6.0 & 0.05 & 120.0 & 2.91 & 50.0 & 0.8 & MXD \\
\hline
\end{tabular}

${ }^{a}$ Predicted ignition regime based on the combustion theory developed and presented by Im et al. [29]. 


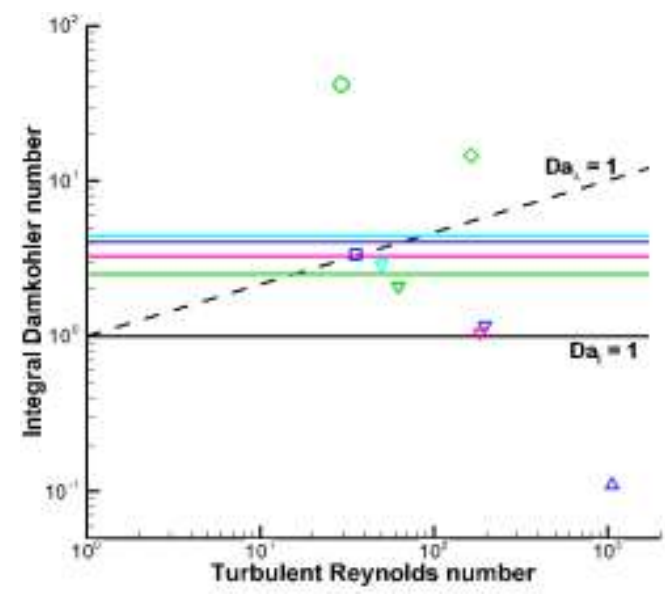

Fig. 2. Parametric cases A (blue square), B (green circle), C (blue triangle), D (green diamond), E (pink delta), F (green diamond), $\mathrm{G}$ (blue delta) and $\mathrm{H}$ (sky blue delta) on the ignition regime diagram. The solid lines with the same colors as the symbols correspond to the respective $\mathrm{Sa}=1$ lines for those cases.

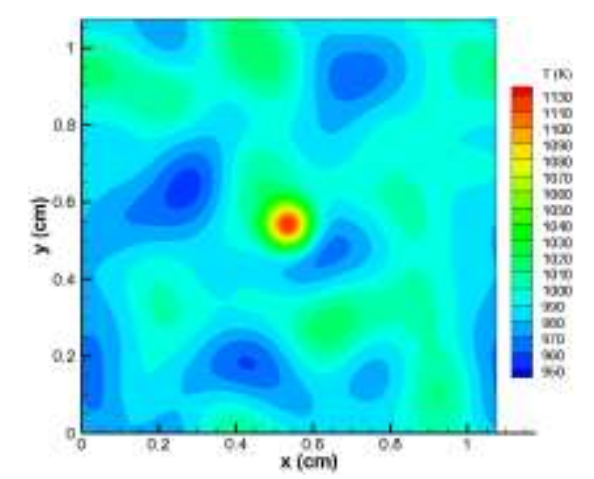

Fig. 3. Initial temperature field for case A.

\section{Results and discussion}

\subsection{General description of auto-ignition phenomena}

For the simulation cases, auto-ignition progress was investigated by monitoring the temporal evolution of the temperature fields, mean pressure and heat release rate. For all cases A$\mathrm{H}$ tested, the observed ignition regimes were found to exhibit the behaviors predicted by the regime diagram as summarized in Table 1 . However, for the sake of brevity, results for only the first three parametric cases A, B and C are reported here which correspond to the $\mathrm{W}, \mathrm{RD}-\mathrm{S}$ and MD-S ignition regimes, respectively. Relative to cases A and C, the initial mean temperature for case $\mathrm{B}$ is higher and case $\mathrm{C}$ has relatively higher turbulence velocity fluctuation as compared to cases A and B. 
Figure 4 show the temporal evolution of temperature field for case A. It is readily observed that a reaction front emanates from the hot spot at the centre of the domain and consumes the majority of the reactant mixture. In contrast, for case B, although there is initially some reaction front propagation from the hot spot (Fig. 5a), the front is not able to consume much of the reactant mixture. Instead, isolated ignition fronts are formed in the end gas, consuming the rest of the mixture as shown in Fig. 5b. This is attributed to the relatively higher reactivity of the end gas which is a result of the higher initial mean temperature. Lastly, for case $\mathrm{C}$, the stronger turbulence level leads to rapid scalar dissipation of the temperature fluctuations (Fig. 6a). Due to strong turbulent mixing, $T$ decreases from the initial value of $15 \mathrm{~K}$ to a minimum value of $6 \mathrm{~K}$, before any ignition fronts develop. Consequently, the reactant mixture auto-ignites almost homogeneously throughout the domain as seen in Fig. 6 b.

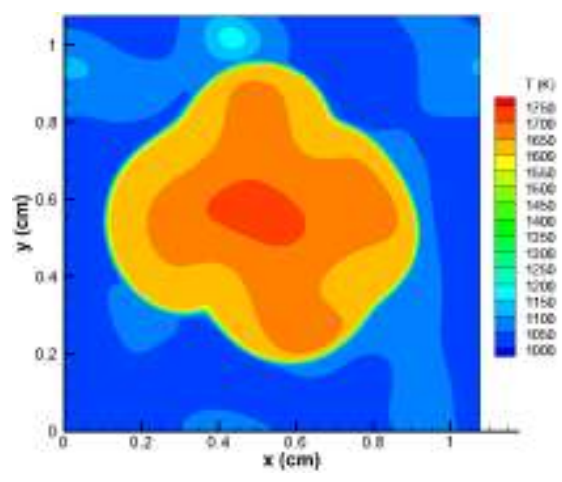

(a)

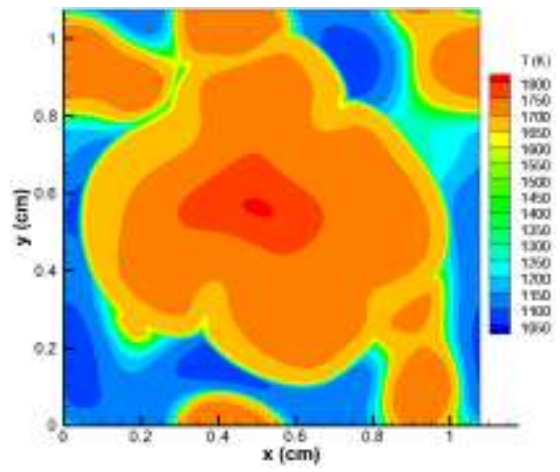

(b)

Fig. 4. Temperature fields for case A at (a) $t / \tau_{i g}=0.41$ and (b) $t / \tau_{i g}=0.48$. 


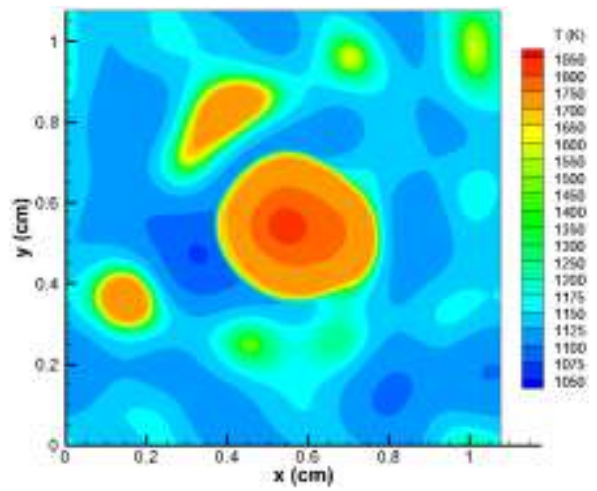

(a)

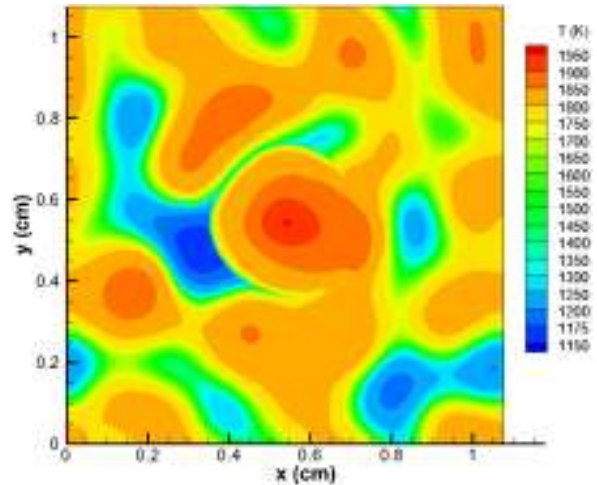

(b)

Fig. 5. Temperature fields for case B at (a) $t / \tau_{i g}=0.63$ and (b) $t / \tau_{i g}=0.77$.

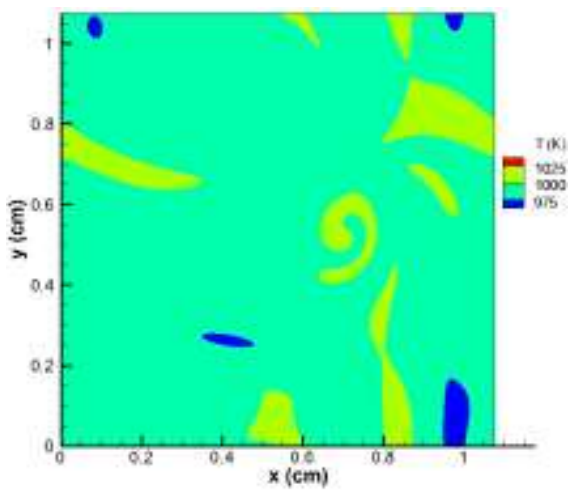

(a) 


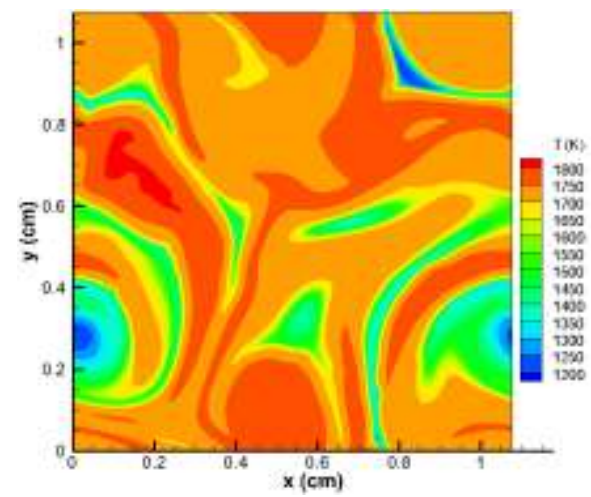

(b)

Fig. 6. Temperature fields for case $\mathrm{C}$ at (a) $t / \tau_{\text {ig }}=0.33$ and (b) $t / \tau_{i g}=0.94$.

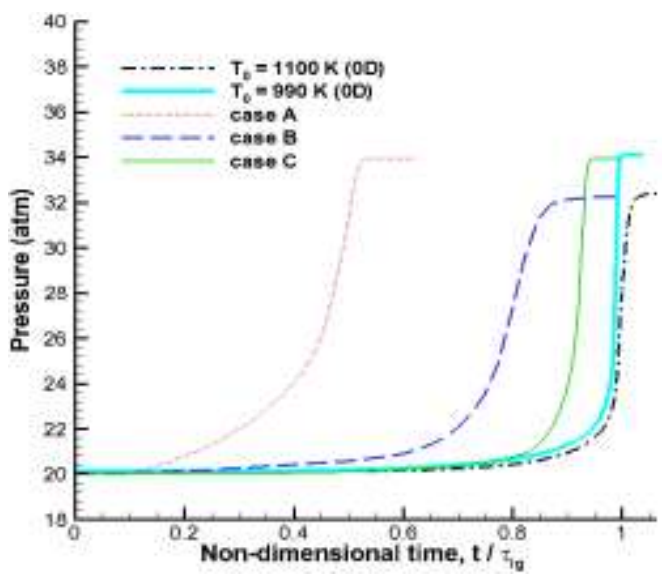

(a)

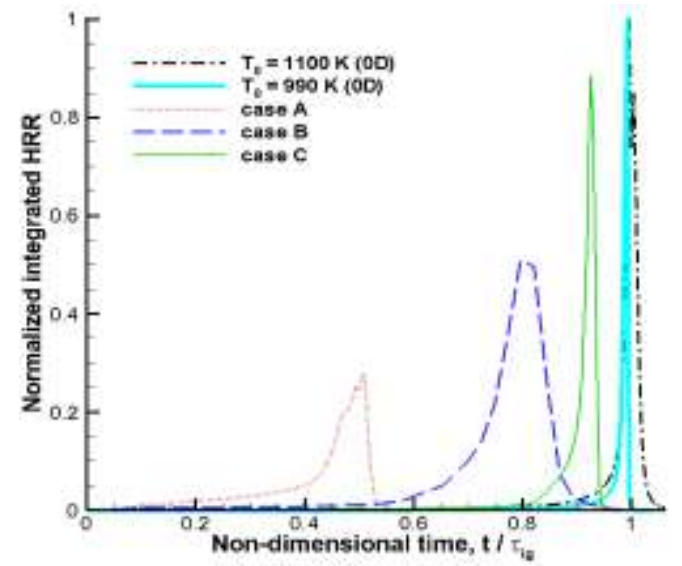

(b)

Fig. 7. Temporal evolution of (a) mean pressure and (b) heat release rate for cases A, B and C. The time is normalized by homogeneous ignition delay time at the mean initial mixture conditions for each case. 
Figure 7 shows the temporal evolution of mean pressure and integrated heat release rate (HRR) for the three cases, where HRR and time are normalized by the maximum integrated HRR and $\tau_{i g}$ of the corresponding zero-dimensional (0D) cases, respectively. For comparison, the temporal evolution of $0 \mathrm{D}$ ignition for $T_{0}=1100 \mathrm{~K}$ and $990 \mathrm{~K}$ are also shown. For case A, the pressure and HRR increase earlier and more slowly than the corresponding 0D case (at $990 \mathrm{~K}$ ), resulting from the deflagrative front propagation. Based on the pressure rise curve for case A, it appears that the fractional contribution of the heat release by deflagration is about half or less, which is in agreement with the regime diagram estimate shown in Fig. 2, where case A is close to the limit between $\mathrm{W}$ and RD-S regimes. The maximum HRR is also much lower. In comparison, cases $\mathrm{B}$ and $\mathrm{C}$ ignite much closer to the corresponding OD ignition delay (at $1100 \mathrm{~K}$ ) and have much higher peak HRRs compared to case A. Moreover, the rates of pressure rise are much higher for cases B and C compared to case A.

\subsection{Front speed analysis}

To characterize the auto-ignition behaviors, front propagation speeds are examined for the cases. The density-weighted front propagation speed [42, 43], $S_{d}$, is employed as a metric to distinguish between deflagration and spontaneous ignition fronts. The isocontour of $Y_{\mathrm{H}_{2}}=0.019$ is chosen to evaluate the mean displacement speed as this particular isocontour coincides approximately with the location of instantaneous maximum HRR, thereby capturing the propagating reaction fronts. A similar approach was used in Ref. [21], and it was found that different choices of the marker species yielded consistent results.

Figure 8 shows the temporal evolution of the mean front speed, normalized by the corresponding laminar flame speed, for cases A, B and C. The mean front speeds exhibit a characteristic U-shape [44, 45], attributed to the initial thermal runaway in the nascent ignition kernel during the early phase of combustion and final stage of fuel consumption. For case A, the

minimum front speed is close to $S_{L}$ (within a factor of 1.5) indicating deflagrative front 
propagation. On the other hand, for cases $\mathrm{B}$ and $\mathrm{C}$, the minimum front speed is much higher (by over a factor of 4) than $S_{L}$, thereby suggesting that spontaneous propagation is the dominant combustion mode. Moreover, the duration of minimum front speed is much shorter for case C, indicating that the mixture auto-ignites nearly homogeneously in this case. These results along with the qualitative description of the ignition progress in section 4.1 suggest that the autoignition behaviors of cases $\mathrm{A}, \mathrm{B}$ and $\mathrm{C}$ are in good agreement with $\mathrm{W}, \mathrm{RD}-\mathrm{S}$ and $\mathrm{MD}-\mathrm{S}$ regimes, respectively, as predicted by the regime diagram.

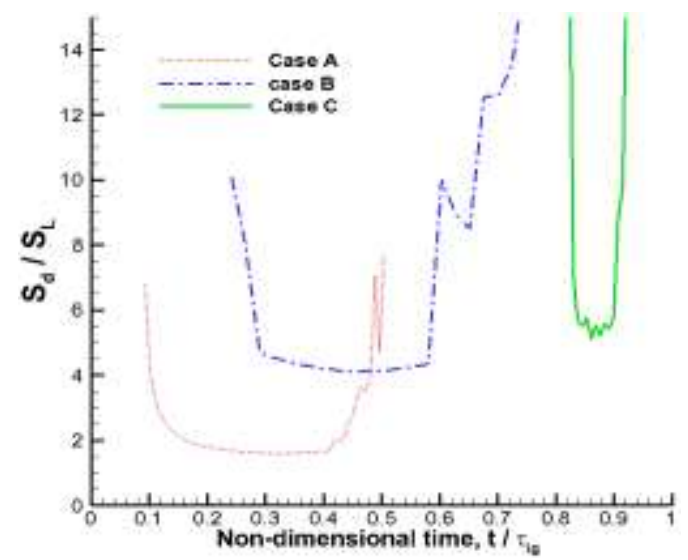

Fig. 8. Temporal evolution of mean front speed for cases A, B and C. The time is normalized by homogeneous ignition delay time at the mean initial mixture conditions for each case.

\subsection{Computational singular perturbation diagnostics}

To gain further insights into the ignition characteristics, a CSP analysis [46, 47, 48] was employed as an automated diagnostic tool to investigate the nature of the local reaction front propagation. CSP allows projection of all relevant chemical and transport processes onto a curvilinear frame of reference spanned by the right eigenvectors of the Jacobian matrix $(d \mathbf{g} / d \mathbf{Z})$ of the chemical source term vector $(\mathbf{g})$, where $\mathbf{Z}$ is the scalar solution variable vector $\left(N_{s}\right.$ species and temperature). The local eigenmodes, equal to the total number of scalar variables $\left(N_{s}+1\right)$, are thus identified having distinct characteristic timescales. Moreover, importance indices can be identified as a non-dimensional measure of the relative contribution of an individual process to the dynamics of a target observable [49, 50]. To assess the role of transport with respect to 
chemistry in ignition front propagation, the importance index of transport (convection and diffusion) to the slow dynamics of temperature, $I^{T}$, is analyzed in the regions ahead of the reaction fronts [50]. By definition, $I^{T}$ ranges from 0 to 1 ; transport prevails over kinetics when this index assumes values close to unity.

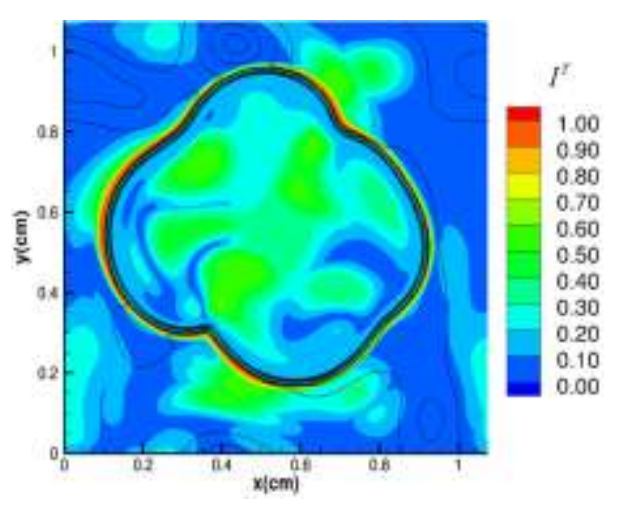

(a)

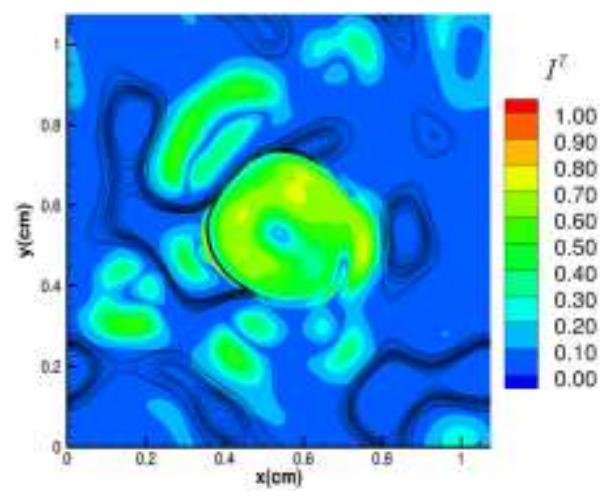

(b)

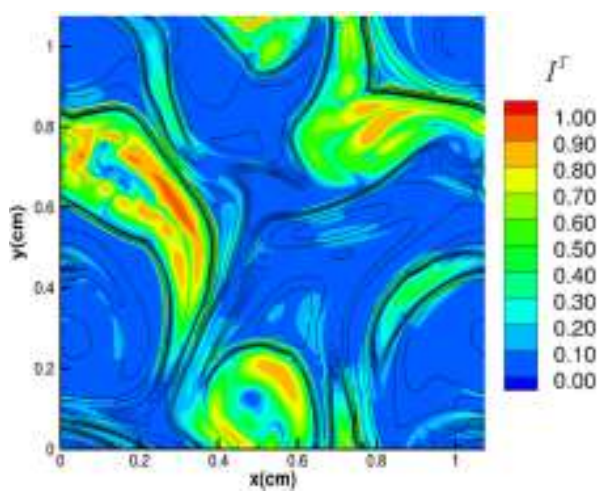

(c)

Fig. 9. $I^{T}$ isocontours for (a) case A $\left(t / \tau_{i g}=0.41\right)$, (b) case B $\left(t / \tau_{i g}=0.77\right)$ and (c) case $\mathrm{C}\left(t / \tau_{i g}=0.94\right)$. The HCO mass fraction contours are overlaid (in black). 
Figure 9a shows the isocontours of $I^{T}$ for case A at $t / \tau_{i g}=0.41$. The corresponding temperature isocontours are shown in Fig. 4a. In addition, isocontours of HCO radical mass fraction (in black) are also superimposed to denote the active reaction zones. This approach was found to be consistent with the identification of upstream regions ahead of the reaction fronts based on the number of exhausted modes (M) [50]. It is clearly seen that $I^{T}$ approaches 1 in the upstream pre-heat region of the front, indicating that the reaction zone propagates upstream by virtue of transport, i.e., as a deflagration front. This confirms that the ignition behavior in this case is indeed in the weak (W) regime. Similarly, Figs. $9 \mathrm{~b}$ and $9 \mathrm{c}$ show the $I^{T}$ and HCO mass fraction isocontours for cases $\mathrm{B}$ and $\mathrm{C}$, at the same time instants for which the temperature profiles are shown in Figs. $5 \mathrm{~b}$ and $6 \mathrm{~b}$, respectively. In contrast to case $\mathrm{A}, I^{T}$ is much lower (< $0.5)$ in the regions upstream of the ignition fronts. Therefore, front propagation is primarily driven by spontaneous ignition in these cases. Combining with the observations from the evolution of the respective temperature fields in Figs. 5 and 6, it can be inferred that the cases B and $\mathrm{C}$ fall into the reaction-dominant and mixing-dominant strong ignition regimes, respectively. The results demonstrate that the ignition regime diagram is able to predict the strong and weak ignition regimes at various parametric conditions of initial turbulent velocity and temperature fluctuations with good fidelity.

\section{Conclusions}

In the present work, 2D DNS of auto-ignition in a uniform syngas/air mixture in the presence of turbulence and temperature fluctuations were performed, at high-pressure lowtemperature conditions. Parametric cases were considered by varying the characteristic integral Damköhler and Reynolds numbers of the system, corresponding to different conditions on the ignition regime diagram of $\mathrm{Im}$ et al. [29]. For all cases, the observed ignition behaviors were found to agree with the predictions of the regime diagram consistently. Three representative cases 
$\mathrm{A}, \mathrm{B}$ and $\mathrm{C}$ were analyzed in detail. Temporal evolution of the temperature field, mean pressure and integrated HRR showed increased propensity for reaction front propagation from a hot spot in case $\mathrm{A}$; whereas for case $\mathrm{B}$, isolated ignition kernels were formed; and for case $\mathrm{C}$, rapid dissipation of the temperature fluctuations due to strong turbulent mixing was readily observed.

Further investigation of the ignition front characteristics was carried out using front speed and CSP importance index analyses. These additional diagnostics consistently showed that ignition in case $\mathrm{A}$ was driven by deflagration, whereas cases $\mathrm{B}$ and $\mathrm{C}$ were dominated by spontaneous ignition. These findings along with the above qualitative description of the ignition phenomena confirmed that the cases could be classified as weak (case A), reaction-dominant strong (case B) and mixing-dominant strong ignition (case C). These results are new and importantly demonstrate the large range of auto-ignition behaviors that can be encountered at low-temperatures and high-pressures for syngas, as well as the profound effects of turbulence and temperature fluctuations to accelerate ignition relative to $0 \mathrm{D}$ reference conditions. Larger advancement of overall ignition and significantly lower HRR relative to homogeneous conditions was observed in the weak ignition regime, in agreement with previous experimental observations [13]. Furthermore, the DNS results were consistent with predictions from the regime diagram based on theoretical scaling analysis. This demonstrates that the physical and chemical mechanisms controlling auto-ignition phenomena in thermally inhomogeneous turbulent reacting flows are well captured by the proposed ignition criteria by Im et al. [29].

Some of the current related ongoing efforts are focused on extending the applicability of the regime diagram to capture detonation by incorporating the effects of acoustics. This will have important implications in the prediction of engine super-knock phenomena. Experimental validation of the ignition regime characterization in compression ignition engines is also underway. 


\section{Acknowledgments}

The authors acknowledge the financial support of U.S. DOE via NETL award number DEFE0007465 and King Abdullah University of Science and Technology (KAUST). This research made use of the computer clusters at KAUST.

\section{References}

[1] J.E. Dec, Proc. Combust. Inst. 32 (2009) 2727-2742.

[2] G.A., Richards, M.M. McMillian, R.S. Gemmen, W.A. Rogers, S.R. Cully, Prog. Energ. Combust. Sci. 27(2) (2001) 141-169.

[3] T. Lieuwen, V. McDonell, D. Santavicca, and T. SattelMayer, Combust. Sci. Technol. 180(6) (2008) 1169-1192.

[4] J.A. Eng, SAE Trans. paper 2002-01-2859, 2002.

[5] V.V. Voevodsky, R.I. Soloukhin, Proc. Combust. Inst. 10 (1965) 279-283.

[6] J.W. Meyer, A.K. Oppenheim, Proc. Combust. Inst. 13 (1971) 1153-1164.

[7] E.S. Oran, T.R. Young, J.P. Boris, A. Cohen, Combust. Flame 48 (1982) 135-148.

[8] E.S. Oran, J.P. Borris, Combust. Flame 48 (1982) 149-161.

[9] R. Blumenthal, K. Fieweger, K.H. Comp, Proc. Int. Symp. Shock Waves 20 (1995), pp. $935-$ 940.

[10] D.J. Vermeer, A.K. Oppenheim, Combust. Flame 18 (1972) 327-336.

[11] K. Fieweger, R. Blumenthal, G. Adomeit, Symp. Combust. 25 (1994) 1579-1585.

[12] S.M. Walton, X. He, B.T. Zigler, M.S. Wooldridge, Proc. Combust. Inst. 31 (2007) 31473154.

[13] A.B. Mansfield, M.S. Wooldridge, Combust. Flame 161 (9) (2014) 2242-2251.

[14] E.L. Peterson, D.M. Kalitan, A.B. Barrett, S.C. Reehal, J.D. Mertens, D.J. Beerer, R.L. Hack, V.G. McDonell, Combust. Flame 149 (2007) 244-247.

[15] C-J. Sung, C.K. Law, Combust. Sci. Technol. 180 (2008) 1097-1116.

[16] F.L. Dryer, M. Chaos, Combust. Flame 152 (2008) 293-299.

[17] M. Chaos, F.L. Dryer, Combust. Sci. Technol. 180 (2008) 1053-1096.

[18] D.J. Beerer, V.G. McDonell, J. Eng. Gas Turb. Power 130 (5): 051507 (2008).

[19] R. Sankaran, H.G. Im, E.R. Hawkes, J.H. Chen, Proc. Combust. Inst. 30 (2005) 875-882.

[20] Y.B. Zeldovich, Combust. Flame 39 (1980) 211-214.

[21] P. Pal, A.B. Mansfield, P.G. Arias, M.S. Wooldridge, H.G. Im, Combust. Theor. Modell. 19 (5) (2015) 587-601.

[22] M. Ihme, Combust. Flame 159 (2012) 1592-1604.

[23] H. Wu, M. Ihme, Combust. Flame 161 (2014) 2317-2326.

[24] C.S. Yoo, T. Lu, J.H. Chen, C.K. Law, Combust. Flame 158 (2011) 1727-1741.

[25] C.S. Yoo, Z. Luo, T. Lu, H. Kim, J.H. Chen, Proc. Combust. Inst. 34 (2013) 2985-2993.

[26] H.A. El-Asrag, Y. Ju, Combust. Flame 161 (2014) 256-269.

[27] S.O. Kim, M.B. Luong, J.H. Chen, C.S. Yoo, Combust. Flame 162 (2015) 717-726.

[28] G. Bansal, A. Mascarenhas, J.H. Chen, Combust. Flame 162 (2015) 688-702.

[29] H.G. Im, P. Pal, M.S. Wooldridge, A.B. Mansfield, Combust. Sci. Technol. 187 (8) (2015) 1263-1275.

[30] K.P. Grogan, S.S. Goldsborough, M. Ihme, Combust. Flame, 162 (2015) 3071-3080. 
[31] J.H. Chen, A. Choudhary, B. de Supinski, M. DeVries, E.R. Hawkes, S. Klasky, W.K. Liao, K.L. Ma, J. Mellor-Crummey, N. Podhorszki, R. Sankaran, S. Shende, C.S. Yoo, Comput. Sci. Disc. 2 (2009) 015001.

[32] C.A. Kennedy, M.H. Carpenter, Appl. Numer. Math. 14 (1994) 397-433.

[33] J. Li, Z. Zhao, A. Kazakov, M. Chaos, F.L. Dryer, J.J. Scire, Int. J. Chem. Kinet. 39 (2007) 109-136.

[34] R.J. Kee, F.M. Rupley, E. Meeks, J.A. Miller, CHEMKIN-III: a fortran chemical kinetic package for the analysis of gas-phase chemical and plasma kinetics, Report No. SAND968216, Sandia National Laboratories, 1996.

[35] R.J. Kee, G. Dixon-Lewis, J. Warnatz, M.E. Coltrin, J.A. Miller, A fortran computer code package for the evaluation of gas-phase multicomponent transport properties, Report No. SAND86-8246, Sandia National Laboratories, 1986.

[36] X.J. Gu, D.R. Emerson, D. Bradley, Combust. Flame 133 (2003) 63-74.

[37] J.H. Chen, S.D. Mason, J.C. Hewson, Proc. Third Joint Sections Meeting of the U.S. Sections of the Combustion Institute, 2003.

[38] J.O. Hinze, Turbulence, McGraw-Hill, New York, 1975.

[39] G. Mittal, C.-J. Sung, Combust. Flame 145 (2006) 160-180.

[40] R.J. Kee, J.F. Grcar, M.D. Smooke, J.A. Miller, A fortran program for modeling steady laminar one-dimensional flames, Report No. SAND85-8240, Sandia National Laboratories, 1985.

[41] S.B. Pope, Turbulent Flows, Cambridge University Press, 2000.

[42] T. Echekki, J.H. Chen, Combust. Flame 118 (1999) 308-311.

[43] H.G. Im, J.H. Chen, Combust. Flame 119 (1999) 436-454.

[44] J.H. Chen, E.R. Hawkes, R. Sankaran, S.D. Mason, H.G. Im, Combust. Flame 145 (2006) 128-144.

[45] E.R. Hawkes, R. Sankaran, P.P. Pebay, J.H. Chen, Combust. Flame 145 (2006) 145-159.

[46] S.H. Lam, Corrado Casci (Ed.), Recent advances in the Aerospace Sciences, Plenum Press, New York and London, 1985, p. 3-20.

[47] S.H. Lam, D.A. Goussis, Twenty-Second Symposium (International) on Combustion (1988) 931-941. Combust. Flame 145 (2006) 145-159.

[48] M. Valorani, H.N. Najm, D.A. Goussis, Combust. Flame 134 (2003) 35-53.

[49] M. Valorani, F. Creta, D.A. Goussis, J.C. Lee, H.N. Najm, Combust. Flame 146 (2006) 2951.

[50] S. Gupta, H.G. Im, M. Valorani, Proc. Combust. Inst. 33 (2011) 2991-2999. 


\section{FIGURE CAPTIONS}

Figure 1 Regime diagram for auto-ignition of homogeneous reactant mixture with temperature fluctuations and turbulence [29].

Figure 2 . Parametric cases A (blue square), B (green circle), C (blue triangle), D (green diamond), $\mathrm{E}$ (pink delta), F (green diamond), G (blue delta) and $\mathrm{H}$ (sky blue delta) on the ignition regime diagram. The solid lines with the same colors as the symbols correspond to the respective $\mathrm{Sa}=1$ lines for those cases.

Figure 3. Initial temperature field for case A.

Figure 4 Temperature fields for case A at (a) $t / \tau_{i g}=0.41$ and (b) $t / \tau_{i g}=0.48$.

Figure 5 Temperature fields for case B at (a) $t / \tau_{i g}=0.63$ and (b) $t / \tau_{i g}=0.77$.

Figure 6 Temperature fields for case $\mathrm{C}$ at (a) $t / \tau_{i g}=0.33$ and (b) $t / \tau_{i g}=0.94$.

Figure 7. Temporal evolution of (a) mean pressure and (b) heat release rate for cases $\mathrm{A}, \mathrm{B}$ and $\mathrm{C}$. The time is normalized by homogeneous ignition delay time at the mean initial mixture conditions for each case.

Figure 8 . ..Temporal evolution of mean front speed for cases A, B and C. The time is normalized by homogeneous ignition delay time at the mean initial mixture conditions for each case.

Figure 9 $I^{T}$ isocontours for (a) case A $\left(t / \tau_{i g}=0.41\right)$, (b) case B $\left(t / \tau_{i g}=0.77\right)$ and (c) case $\mathrm{C}\left(t / \tau_{i g}=0.94\right)$. The HCO mass fraction contours are overlaid (in black). 